\title{
Pre-Pandemic Peer Relations Predict Adolescents' Internalizing Response to Covid-19
}

\author{
Fanny Mlawer ${ }^{1} \cdot$ Christina C. Moore $^{1} \cdot$ Julie A. Hubbard ${ }^{1}$ (D) $\cdot$ Zachary M. Meehan $^{1}$ \\ Accepted: 18 October 2021 / Published online: 26 October 2021 \\ (C) The Author(s), under exclusive licence to Springer Science+Business Media, LLC, part of Springer Nature 2021
}

\begin{abstract}
The goal of the current longitudinal study was to investigate the role of adolescents' peer victimization and aggression prior to COVID-19 on the change in their depressive and anxious symptoms from pre- to mid-pandemic. We hypothesized that, although adolescents overall would display an increase in internalizing symptoms from pre- to mid-pandemic, this response would be weakened or perhaps even reversed when adolescents experienced high levels of victimization or aggression prior to the pandemic. Participants included 96 racially/ethnically diverse adolescents ( 42 males, 53 females; 1 other) with an average age of 16.79 years $(S D=0.60)$. At Time 1 (T1; June 2019 through February 2020; pre-pandemic), adolescents completed self-report measures of their peer relations (aggression, victimization) and internalizing symptoms (depressive, anxious). At Time 2 (T2; May through July 2020; mid-pandemic), adolescents completed self-report measures of their internalizing symptoms (depressive, anxious). On average, adolescents' anxious and depressive symptoms increased from T1 to T2, although they exhibited substantial variability, with reports ranging from decreasing symptoms to increasing symptoms. Although on average adolescents reported increases in anxious symptoms from T1 to T2, adolescents with higher T1 peer victimization reported less positive change in anxious symptoms. Similarly, although on average adolescents reported increases in depressive symptoms from $\mathrm{T} 1$ to $\mathrm{T} 2$, adolescents with higher levels of $\mathrm{T} 1$ aggression reported less positive change in depressive symptoms from T1 to T2. Discussion focused on restrictions on in-person peer interactions necessitated by COVID-19 that may reduce adolescents' distress when their pre-pandemic daily lives were characterized by negative peer relations.
\end{abstract}

Keywords Peer victimization · Aggression · Depressive symptoms · Anxious symptoms · COVID-19

The COVID-19 pandemic has posed unprecedented and unique challenges for people from all walks of life. Many regions have implemented strict public health regulations, including social distancing, to limit the spread of the virus. For most adolescents, these restrictions mean decreasing and limited in-person social interaction (Gadassi Polack et al., 2021), often including a transition to online school (Malkus $\&$ Christensen, 2020). Historically, similar disease containment measures, including quarantine and isolation, have had a negative impact on psychological well-being, including increases in both the prevalence and intensity of internalizing symptoms (e.g., Hossain et al, 2020). Given the importance of peer relationships in adolescence (e.g., Bagwell \& Bukowski, 2018), as well as adolescents' increased risk for

Julie A. Hubbard

jhubbard@udel.edu

1 Department of Psychological and Brain Sciences, University of Delaware, Newark, DE, US internalizing disorders in comparison to adults (e.g., Adkins et al., 2009), the social restrictions accompanying COVID19 seem especially likely to lead to increased internalizing distress in adolescents (Beam \& Kim, 2020; Rodman et al., 2021).

In fact, parents and teens report adverse mental health effects of the pandemic on adolescents (e.g., Magklara et al., 2020) including anxious and depressive symptoms (e.g., Xie et al., 2020; Zhou et al., 2020). Moreover, adolescents who are lonelier (Ellis et al., 2020; Liu et al., 2020), have less social support (Qi et al., 2020; Rodman et al., 2021), and spend less time with peers either in-person or online (Rodman et al., 2021) report greater depressive and/ or anxious symptoms during COVID-19. These findings are consistent with broader literature linking adolescent loneliness and isolation to internalizing symptoms, including self-harm and suicidal ideation, both concurrently and longitudinally (Hall-Lande et al., 2007; Loades et al., 2020; Qualter \& Munn, 2002). 
The COVID studies reviewed above suggest that, for most adolescents, reductions in face-to-face interactions with peers during the pandemic likely have contributed to feelings of loneliness and isolation as well as concomitant increases in internalizing symptoms. In contrast, the picture may look quite different for adolescents who experienced particularly challenging peer relations such as aggression toward peers (Vitaro et al., 2018) or victimization from peers (Salmivalli \& Peets, 2018) prior to the start of the pandemic. In pre-pandemic literature, peer victimization and aggression are linked to internalizing symptoms concurrently, longitudinally, and at the daily level (e.g., Gini et al., 2018; Hawker \& Boulton, 2000; Rothenberg et al., 2019), with victimization linked to both depressive and anxious symptoms (Adrian et al., 2019; Forbes et al., 2019; Vuijk et al., 2007) and aggression linked more tightly to depressive than anxious symptoms (Blain-Arcaro \& Vaillancourt, 2017; Dutton \& Karakanta, 2013; Gresham et al., 2016). However, high levels of pre-pandemic peer victimization or aggression may actually lessen normative increases in depressive and anxious symptoms during COVID-19 by reducing adolescents' exposure to aversive peer interactions through restrictions on social contact necessitated by the pandemic.

Empirical support for this prediction is based in three lines of reasoning. First, victimized and aggressive adolescents sometimes withdraw from peers (e.g., McDougall \& Vaillancourt, 2015; Rubin et al., 2018), perhaps in part to avoid the depressive and anxious feelings that difficult peer interactions generate. Second, longitudinal studies suggest that decreases in adolescents' displays of aggression and experiences of victimization are accompanied by concomitant decreases in internalizing symptoms (Blain-Arcaro \& Vaillancourt, 2017; Gini et al., 2018; Reijntjes et al., 2010). Finally, a recent study found that adolescents experienced fewer internalizing symptoms when their reason for quarantining during COVID-19 was that they preferred to stay at home, rather than to avoid infection or judgment from others (Oosterhoff et al., 2020). Taken together, this work suggests that, for those adolescents whose pre-pandemic daily lives were characterized by peer victimization or aggression, the social restrictions of COVID-19 may help alleviate internalizing symptoms associated with these negative peer interactions.

Thus, the goal of the current longitudinal study was to investigate the role of adolescents' peer victimization and aggression prior to the pandemic on their change in depressive and anxious symptoms from pre- to mid-pandemic. Our hypothesis was that, although adolescents overall would display an increase in internalizing symptom response from preto mid-pandemic, this response would be weakened or perhaps even reversed when adolescents experienced high levels of peer victimization or aggression prior to the pandemic.

\section{Method}

At Time 1 (T1; June 2019 through February 2020; prepandemic), we collected self-report data from a racially/ ethnically diverse sample of adolescents on their peer relations (aggression, victimization) and internalizing symptoms (depressive, anxious). At Time 2 (T2; May through July 2020; mid-pandemic), we collected self-report data from this same sample on their internalizing symptoms (depressive, anxious). The average number of months between $\mathrm{T} 1$ and $\mathrm{T} 2$ was $9.08(S D=1.90)$. The Institutional Review Board of the University of Delaware approved the project.

\section{Participants}

The time points we refer to as $\mathrm{T} 1$ and $\mathrm{T} 2$ were the fourth and fifth times we collected data from these participants. The original cohort was recruited during the 2013-2014 academic year from 74 th- and 5th-grade classrooms in 9 elementary schools in one school district in a mid-Atlantic state. We sent home parental permission forms with 1,910 children, $62 \%$ of these children received both parental consent and child assent and completed data collection $(N=1191)$, and 988 parents' provided permission to be re-contacted for future studies.

Time 1 At T1, we recruited a subsample of the original cohort through mail, email, and phone contacts for additional data collection when they were in 10th or 11th grade ( $N=147 ; 68$ males, 78 females, 1 other). We contacted all participants with up-to-date contact information who could be reached, and we recruited all participants whose parents consented and who assented, resulting in a contacted-andconsented rate of $15 \%$ six years after initial data collection. The racial/ethnic breakdown of this sample was $65 \%$ European American, 12\% African American, 10\% Latino American, $7 \%$ Asian American, and 6\% of mixed race or ethnicity. The average age of participants at $\mathrm{T} 1$ was 16.02 $(S D=0.63)$. Parents reported annual household income as less than $\$ 20,000(3 \%)$, $\$ 20,000-\$ 50,000$ (16\%), \$50,000$100,000(22 \%), \$ 100,000-\$ 150,000(22 \%)$, and greater than $\$ 150,000$ (36\%). Families who verbally agreed to participate scheduled a 2-hour lab visit during which we obtained parental consent and adolescent assent.

We compared the 147 adolescents who completed data collection at $\mathrm{T} 1$ to the 1044 adolescents in the original cohort who did not complete data collection at $\mathrm{T} 1$ on demographic variables as well as victimization (teacher, self-, and peer-report), aggression (teacher- and peerreport), depressive symptoms (teacher- and self-report), 
and anxious symptoms (teacher- and self-report) at the time of original data collection. The only differences to emerge were that: a) youth included in the T1 data collection $(64 \%)$ were more likely to be European American than youth who did not participate at T1 $(50 \%), \chi^{2}(1)=10.07$, $p<0.01$, and b) youth included in the $\mathrm{T} 1$ data collection $($ mean $=0.04)$ were rated lower by peers on aggression at the time of original data collection than youth who did not participate at $\mathrm{T} 1($ mean $=0.07), F(1,1189)=7.26, p<0.01$.

Time 2 At T2, we recruited as many of the T1 cohort as possible for additional data collection. The resulting sample included 96 adolescents (42 males, 53 females; 1 other). The racial/ethnic breakdown of this sample was 69\% European American, 10\% Latino American, 8\% African American, $7 \%$ Asian American, and $6 \%$ of mixed race or ethnicity. The average age of participants at T2 was $16.79(S D=0.60)$. Parents and adolescents provided online consent/assent, and then adolescents completed an online questionnaire. Of the $147 \mathrm{~T} 1$ participants, 43 parents (29\%) refused consent or did not respond, 8 adolescents (6\%) refused assent, and 96 adolescents (65\%) participated.

We compared the 96 adolescents who completed data collection at both $\mathrm{T} 1$ and $\mathrm{T} 2$ to the 51 adolescents who completed data collection at $\mathrm{T} 1$ only on demographic variables and each of the four T1 study variables described below. The only difference to emerge was that T1-T2 participants $($ mean $=2.14)$ were more anxious at $\mathrm{T} 1$ than T1-only participants $($ mean $=1.97), F(1,145)=4.95, p<0.05$.

\section{Procedures}

Time 1 During a two-hour lab visit that included additional measures as part of a larger study, adolescents completed the measures described below on a tablet or computer. We compensated parents (\$10) and adolescents (\$25) at the end of the visit.

Time 2 Adolescents completed the measures described below via a five-minute online Qualtrics survey. We compensated adolescents with a $\$ 5$ Amazon electronic gift card.

\section{Measures}

Victimization We assessed victimization at $\mathrm{T} 1$ via self report using the 20-item Forms of Peer Victimization Scale (FPVS; Morrow et al., 2021) with a response scale ranging from $1=$ not at all to $5=a$ whole lot. A sample item was "A kid hit or pushed me," and participants responded regarding the extent of their victimization "recently." Although the measure does not explicitly mention online victimization, many of the items are worded so as to apply to both face-to-face and online contexts (e.g., "A kid called me mean names," "A kid tried to make my friends turn against me"). We averaged across all items to create the variable T1 Victimization, with higher scores reflecting greater victimization. Cronbach's alpha was 0.90 at T1.

Aggression We assessed aggression at $\mathrm{T} 1$ using the 36-item Forms and Functions of Aggression Questionnaire (FFAQ; Little et al., 2003) with a response scale ranging from $1=$ not at all true to $4=$ completely true. A sample item was "I'm the kind of person who often fights with others." We averaged across all 36 items to create an assessment of overall T1 Aggression, with higher scores reflecting greater aggression. Cronbach's alpha at T1 was 0.89 .

Depressive Symptoms We assessed depressive symptoms at T1 and T2 using the 12-item Children's Depression Inventory 2 Self-Report Short Version (Kovacs, 2011). For each item, participants marked one of three statements best describing their feelings within the past two weeks. A sample item included the statements "I am sad once in a while," "I am sad many times," and "I am sad all the time," and the response scale ranged from $1=$ low level of symptom to $3=$ high level of symptom. Cronbach's alpha was 0.82 at both time points. Items were averaged to create the variables T1 (T2) Depressive Symptoms, with higher scores reflecting greater depressive symptoms.

Anxious Symptoms We measured anxious symptoms at T1 and T2 using the Multidimensional Anxiety Scale for Children (MASC; March et al., 1997). At T1, we used the full 39-item measure, and at T2, we used the shortened 10-item measure (MASC-10; March et al., 1997). A sample item was "I feel restless or on edge," and adolescents responded on a scale from $1=$ never true about me to $4=$ often true about me. Items were reverse-scored as needed and then averaged to create the variables T1 (T2) Anxious Symptoms, with higher scores reflecting greater anxious symptoms. Cronbach's alpha was 0.92 at T1 and 0.83 at T2.

\section{Data Analysis Plan}

First, we conducted preliminary analyses including means, standard deviations, ranges, correlations, and assessment of possible demographic covariates. Next, we examined changes in depressive and anxious symptoms from $\mathrm{T} 1$ to T2. Finally, we used structural equation modeling to assess the impact of T1 Victimization and Aggression on changes in adolescents' anxious and depressive symptoms from $\mathrm{T} 1$ to T2. Analyses were conducted in Mplus version 8 using robust maximum likelihood estimating to account for variable skew (Muthén \& Muthén, 1998-2017). 


\section{Results}

\section{Preliminary Analyses}

Means, standard deviations, ranges, and bivariate correlations are presented in Table 1 . We assessed potential demographic covariates using one-way analysis of variance, with demographic variables (Gender, Race, Household Income) predicting study variables (T1 Peer Victimization, T1 Aggression, T2 Anxious Symptoms, T2 Depressive Symptoms) in 12 separate analyses. Gender $(0=$ females, $1=$ males $)$ was the only demographic variable that was significantly associated with variables of interest. Specifically, girls reported more T2 Anxious Symptoms $(F(1,93)=13.88, p<0.001 ; M=2.39)$ and T2 Depressive Symptoms $(F(1,93)=13.92, p<0.001 ; M=1.60)$ compared to boys $(M \mathrm{~T} 2$ Anxious Symptoms $=1.95 ; M$ T2 Depressive Symptoms $=1.35$ ). Thus, Gender was included as a covariate in primary analyses.

\section{Primary Analyses}

To assess change in adolescents' anxious and depressive symptoms from $\mathrm{T} 1$ to $\mathrm{T} 2$, we used latent change score modeling (Kievit et al., 2018). Latent change scores are conceptualized as the function of autoregressive and residual components. We set the autoregressive path (T2 Anxious/ Depressive Symptoms on T1 Anxious/Depressive Symptoms) to 1 and the T2 Anxious/Depressive Symptoms variance to 0 . The latent change scores were indicated by $\mathrm{T} 2$ Anxious/Depressive Symptoms with a loading fixed to 1, which accounts for all the residual variance in T2 Anxious/ Depressive Symptoms. We allowed T1 indicators to covary with their respective latent change score (e.g., T1 Anxious Symptoms with Anxious Symptom Change) to account for possible associations between adolescents' starting levels of anxious/depressive symptoms and their degree of change. These parameter constraints allow the latent change scores to separate true score change from measurement error (McArdle, 2009). Latent change scores for anxious and depressive symptoms covaried.

To determine the influence of earlier peer relations on changes in adolescents' internalizing symptoms during COVID-19, latent change scores were regressed on T1 Peer Victimization and T1 Aggression. T1 Peer Victimization and T1 Aggression were grand mean centered to aid interpretation. We allowed T1 Peer Victimization and T1 Aggression to covary. Modification indices showed that allowing T1 Peer Victimization and T1 Aggression to covary with T1 Anxious and T1 Depressive Symptoms significantly improved model fit. This final model had excellent model fit $\left(\left(X^{2}(27, N=96)=6.28, p=0.39 ; \mathrm{CFI}=1.00 ; \mathrm{TLI}=1.00\right.\right.$; SRMR $=0.05 ;$ RMSEA $=0.02$ ).

On average, adolescents' reports of anxious and depressive symptoms increased from T1 to T2. Random intercepts for Anxious Symptom Change and Depressive Symptom Change revealed that adolescents exhibited substantial variability in anxious and depressive symptoms from $\mathrm{T} 1$ to $\mathrm{T} 2$, with reports ranging from decreasing rates to increasing rates. More specifically, the range from one (two) $S D s$ below to above the mean was $-0.07(-0.32)$ to $0.41(0.66)$ for Anxious Symptom Change and $-0.02(-0.20)$ to $0.32(0.50)$ for Depressive Symptom Change, with 32 (7) teens falling 1 (2) $S D$ below the mean for Anxious Symptom Change and 41 (24) teens falling 1 (2) $S D$ below the mean for Depressive Symptom Change. Anxious Symptom Change and Depressive Symptom Change were positively correlated

Table 1 Descriptive statistics and bivariate correlations among study variables

\begin{tabular}{|c|c|c|c|c|c|c|c|}
\hline Variable & 1 & 2 & 3 & 4 & 5 & 6 & 7 \\
\hline 1. T1 Peer Victimization & - & & & & & & \\
\hline 2. T1 Aggression & $0.31 *$ & - & & & & & \\
\hline 3. T1 Anxious Symptoms & $0.45^{*}$ & 0.10 & - & & & & \\
\hline 4. T2 Anxious Symptoms & 0.12 & 0.05 & $0.61 *$ & - & & & \\
\hline 5. T1 Depressive Symptoms & $0.54 *$ & $0.42 *$ & $0.63 *$ & $0.37 *$ & - & & \\
\hline 6. T2 Depressive Symptoms & $0.40 *$ & 0.10 & $0.60^{*}$ & $0.60^{*}$ & $0.68 *$ & - & \\
\hline 7. Gender & 0.06 & 0.18 & -0.15 & $-0.30^{*}$ & -0.00 & $-0.34 *$ & - \\
\hline Mean & 1.37 & 1.23 & 2.14 & 2.20 & 1.43 & 1.49 & 0.46 \\
\hline Standard Deviation & 0.45 & 0.25 & 0.47 & 0.60 & 0.33 & 0.34 & 0.52 \\
\hline Range & $1.00-3.55$ & $1.00-2.49$ & $1.36-3.31$ & $1.00-3.60$ & $1.00-2.42$ & $1.00-2.42$ & $0.00-2.00$ \\
\hline Skew & 2.14 & 2.00 & 0.80 & -0.05 & 0.99 & 0.55 & 0.40 \\
\hline \# of Participants $1 S D$ Above Mean & 14 & 14 & 18 & 20 & 17 & 21 & - \\
\hline \# of Participants 2 SD Above Mean & 5 & 4 & 6 & 1 & 4 & 3 & - \\
\hline
\end{tabular}

$\mathrm{T} 1=$ pre-pandemic, $\mathrm{T} 2=$ mid-pandemic $;=96 ; * p<.01$ 
$(r=0.30, p<0.01)$, indicating that adolescents who reported greater increases in anxious symptoms also reported greater increases in depressive symptoms during COVID-19.

Focal to study hypotheses, regression coefficients showed that T1 Peer Victimization was a significant predictor of Anxious Symptom Change, whereas T1 Aggression was a significant predictor of Depressive Symptom Change. More specifically, T1 Peer Victimization negatively predicted Anxious Symptom Change, such that adolescents with more T1 Peer Victimization reported less positive change in anxious symptoms from $\mathrm{T} 1$ to $\mathrm{T} 2$ (Est $=-0.28, p=0.02$ ). To aid interpretation, we ran two post hoc analyses that centered the intercept of Anxious Symptom Change at 1 and 2 SD above the mean on peer victimization to examine the level and significance of Anxious Symptom Change for adolescents at high levels of peer victimization. Although Anxious Symptom Change was positive and significant $(\mathrm{Est}=0.17$, $p<0.05$ ) for adolescents at average levels of T1 Peer Victimization (as described in the paragraph above), Anxious Symptom Change was not significant at $1 \mathrm{SD}(\mathrm{Est}=0.12$, $n s)$ or 2 SDs (Est $=-0.01, n s)$ above the mean. Similarly, T1 Aggression negatively predicted Depressive Symptom
Change, such that adolescents reporting higher T1 Aggression reported less positive change in depressive symptoms from T1 to T2 (Est $=-0.34, p<0.01$ ). Although Depressive Symptom Change was positive and significant $(\mathrm{Est}=0.15$, $p<0.01$ ) for adolescents at average levels of T1 Aggression (as described in the paragraph above), post hoc analyses indicated that Depressive Symptom Change was not significant at $1 \mathrm{SD}(\mathrm{Est}=0.10, n s)$ or $2 \mathrm{SDs}(\mathrm{Est}=0.01, n s)$ above the mean. No significant associations emerged between T1 Peer Victimization and Depressive Symptom Change or T1 Aggression and Anxious Symptom Change. Gender significantly predicted Anxious and Depressive Symptom Change, with boys' reporting significantly less change in internalizing symptoms relative to girls. See Table 2 for parameter estimates and Fig. 1 for the model.

We re-ran these analyses with the addition of two potential timing covariates (length of time between T1 and T2 data collection and length of time between our state's stay-at-home order and T2 data collection). Neither timing covariate changed the pattern of findings, and so for simplicity's sake, we did not include them in the primary analyses reported here.
Table 2 Path model of pre-pandemic peer relations predicting change in anxious and depressive symptoms from pre- to mid-pandemic

\begin{tabular}{|c|c|c|c|c|}
\hline & Estimate & (SE) & $\mathrm{z}$ & $p$ \\
\hline \multicolumn{5}{|l|}{ Intercepts } \\
\hline Change in Anxious Symptoms & 0.17 & 0.06 & 2.66 & 0.01 \\
\hline Change in Depressive Symptoms & 0.15 & 0.03 & 4.81 & $<0.01$ \\
\hline \multicolumn{5}{|l|}{ Change in Anxious Symptoms } \\
\hline T1 Victimization & -0.28 & 0.10 & -2.74 & 0.01 \\
\hline T1 Aggression & -0.06 & 0.17 & -0.36 & 0.72 \\
\hline Gender & -0.22 & 0.09 & -2.44 & 0.02 \\
\hline \multicolumn{5}{|l|}{ Change in Depressive Symptoms } \\
\hline T1 Victimization & -0.03 & 0.07 & -0.40 & 0.69 \\
\hline T1 Aggression & -0.34 & 0.09 & -3.69 & $<0.01$ \\
\hline Gender & -0.19 & 0.05 & -3.99 & $<0.01$ \\
\hline \multicolumn{5}{|l|}{ Covariances } \\
\hline T1 Anxious Symptoms with Change in Anxious Symptoms & -0.03 & 0.01 & -2.39 & 0.02 \\
\hline T1 Depressive Symptoms with Change in Depressive Symptoms & -0.02 & 0.01 & -3.04 & $<0.01$ \\
\hline Change in Anxious Symptoms with Change in Depressive Symptoms & 0.03 & 0.01 & 3.25 & $<0.01$ \\
\hline \multicolumn{5}{|l|}{ Variances } \\
\hline T1 Anxious Symptoms & 0.22 & 0.03 & 6.99 & $<0.01$ \\
\hline T1 Depressive Symptoms & 0.11 & 0.02 & 6.98 & $<0.01$ \\
\hline T1 Victimization & 0.20 & 0.06 & 3.56 & $<0.01$ \\
\hline T1 Aggression & 0.06 & 0.02 & 3.52 & $<0.01$ \\
\hline \multicolumn{5}{|l|}{ Residual Variances } \\
\hline Change in Anxious Symptoms & 0.21 & 0.03 & 6.91 & $<0.01$ \\
\hline Change in Depressive Symptoms & 0.05 & 0.01 & 7.35 & $<0.01$ \\
\hline
\end{tabular}

Estimates are unstandardized 


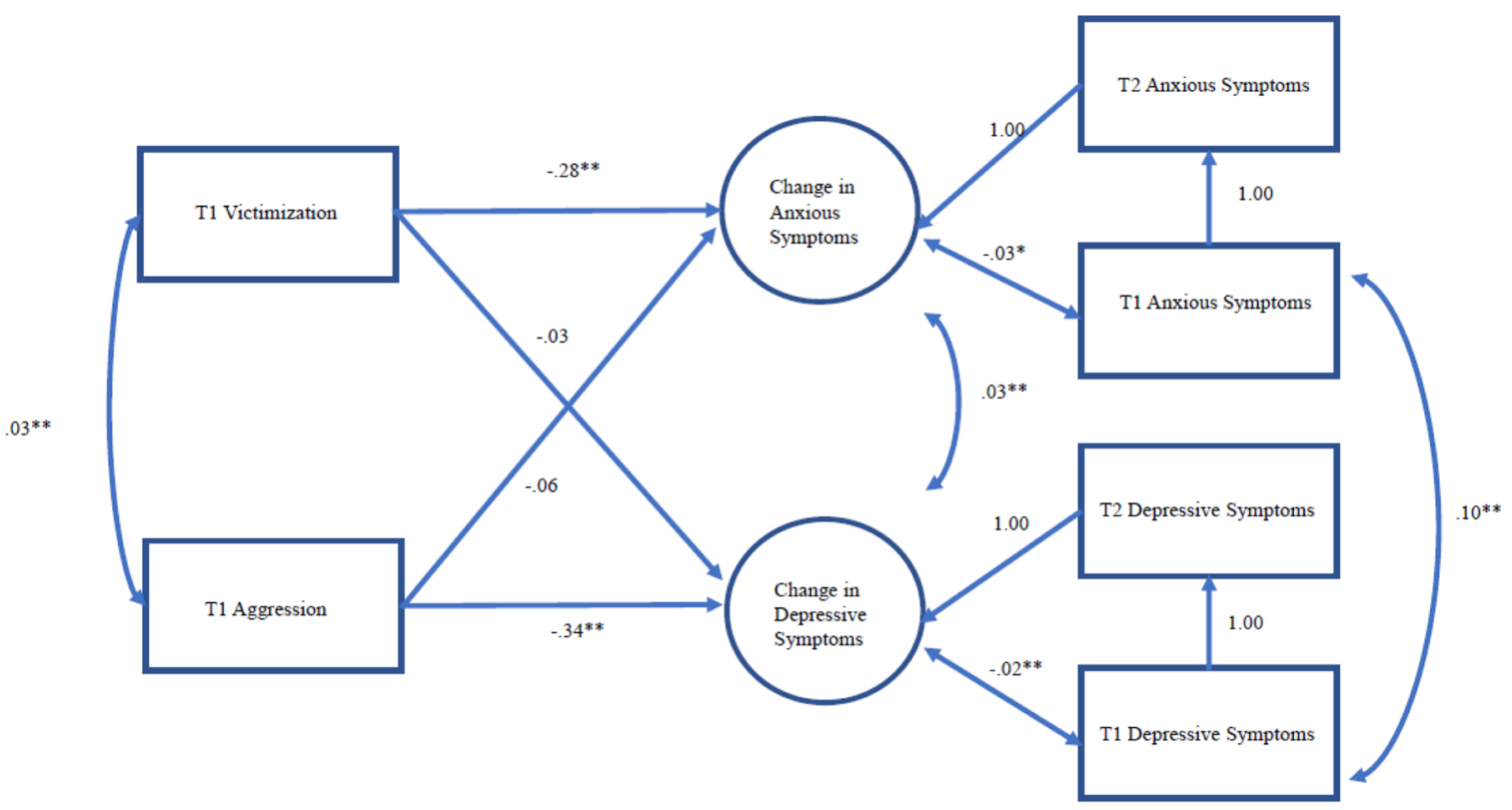

Note. $\mathrm{T} 1=$ pre-pandemic, $\mathrm{T} 2=$ mid-pandemic; $N=96 ;{ }^{*} p<.05,{ }^{* *} p<.01$; estimates are unstandardized.

Not depicted in model: a) Change in Anxious Symptoms covarying with Gender $($ Est $=-.22, p<.05)$, b) Change in Depressive Symptoms covarying with Gender (Est $=-.19, p<.01)$, c) T1 Victimization covarying with T1 Anxious Symptoms $($ Est $=.09, p<$ $.01)$, d) T1 Victimization covarying with T1 Depressive Symptoms (Est $=.08, p<.01)$, e) T1 Aggression covarying with T1 Anxious Symptoms $($ Est $=.01, n s)$, and $\mathrm{f}) \mathrm{T} 1$ Aggression covarying with T1 Depressive Symptoms $($ Est $=.03, p<.01)$.

Fig. 1 Model of pre-pandemic victimization and aggression predicting change in anxious and depressive symptoms from pre- to mid-mandemic

\section{Discussion}

The primary goal of this study was to investigate the role of adolescents' negative peer relations (aggression toward peers, victimization from peers) prior to the pandemic on their change in depressive and anxious symptoms from preto mid-pandemic. We began, though, by simply examining the average trajectory of adolescents' internalizing symptoms from before COVID-19 to the middle of the pandemic. In line with hypotheses, and not surprisingly, adolescents' internalizing symptoms on average increased from pre- to mid-pandemic. This finding is consistent with recent crosssectional studies in which parents and teens reported anxious and depressive symptoms in response to COVID-19 (e.g., Magklara et al., 2020; Xie et al., 2020; Zhou et al., 2020). The current longitudinal study extends these findings by suggesting that adolescents not only experience internalizing symptoms during COVID-19, but in fact, for the average adolescent, these symptoms worsen from pre-pandemic levels (see Gadassi Polack et al., 2021 for a similar finding). This pattern is in contrast to pre-pandemic literature on the normative trajectory of depressive and anxious symptoms, which suggests that symptoms peak in early to mid-adolescence and then remain stable or decline through the late adolescent period that characterizes our sample (Nelemans et al., 2014;
Ohannessian et al., 2017; Schubert et al., 2017). For many adolescents, the social restrictions accompanying COVID19 likely are at least partially responsible for their increased anxious and depressive feelings (Ellis et al., 2020; Liu et al., 2020; Qi et al., 2020), although other factors such as health or economic stress likely also play an important role.

However, all adolescents did not follow this pattern. Instead, they varied widely in the degree and direction of change in internalizing symptoms from pre- to mid-pandemic, with change ranging from increasing to decreasing. Moreover, and also consistent with hypotheses, adolescents who reported high levels of difficult interactions with peers prior to the pandemic reported less change in internalizing symptoms from pre- to mid-pandemic. In other words, negative peer experiences prior to the pandemic lessened the extent to which adolescents experienced increases in internalizing symptoms from pre- to mid-pandemic, with adolescents at high (1 and 2 SD above mean) peer victimization and aggression reporting no significant changes in their anxious and depressive symptoms, respectively. One possible explanation for this finding is that adolescents with poor peer relationships were already more anxious and depressed prior to the pandemic and therefore were not impacted by the loss of face-to-face interactions with friends or classmates. It is also possible that a subset of adolescents with especially challenging peer interactions 
may have viewed the face-to-face social restrictions necessitated by the pandemic in a positive light or as a relief. The fact that some teens may have found respite in COVID-19, in spite of the health and economic challenges the pandemic has wrought, sheds light on just how distressing their prepandemic in-person peer experiences may have been.

At the same time, regardless of the temptation to view the pandemic as a "natural experiment" in limited social interaction, caution is warranted in drawing strong causal connections between the reductions in face-to-face social contact necessitated by COVID-19 and reductions in typical increases in internalizing symptoms. Given that we did not assess participants' peer interactions during the pandemic, we do not know whether their online interactions increased, decreased, or remained stable as their as face-to-face interactions decreased. However, recent longitudinal work on adolescents' peer interactions pre- to mid-pandemic suggests that while overall peer interactions decreased (Gadassi Polack et al., 2021), face-to-face interactions decreased particularly sharply and online interactions remained stable (Rodman et al., 2021). If our participants' online interactions in fact did increase or at least remain stable, did those who engaged in more aggression or experienced more victimization prepandemic continue this pattern mid-pandemic, albeit likely in a largely online context? Or, did they experience overall decreases in their aggression or victimization? The answer to this question is essential to understanding whether the lack of change in internalizing symptoms that we observed for teens with challenging peer relations are linked to decreases in overall peer interaction or face-to-face interaction in particular. To answer this question, future researchers should assess peer relations at all time points in longitudinal COVID-19 studies and include explicit measures of online peer interaction.

Of note, the links between pre-pandemic peer relations and change in internalizing symptoms from pre- to midpandemic were quite specific. Adolescents who reported greater pre-pandemic peer victimization experienced less change in anxious symptoms, and adolescents who reported greater pre-COVID aggression toward peers experienced less change in depressive symptoms. However, prepandemic peer victimization was not linked to change in depressive symptoms, and pre-pandemic aggression was not related to change in anxious symptoms.

In pre-COVID literature, peer victimization has been linked to both anxious and depressive symptoms both concurrently and longitudinally (e.g., Forbes et al., 2019; Hawker $\&$ Boulton, 2000; Vuijk et al., 2007). How then do we explain the specificity of the link between pre-pandemic victimization and change in anxious but not depressive symptoms? One possibility is the mechanisms through which peer victimization acts on future internalizing symptoms, including increased rumination (e.g., Adrian et al., 2019) about future victimization experiences (e.g., Boulton et al., 2008). Because COVID-19 has greatly restricted face-to-face social interactions, adolescents who typically experience high levels of anticipatory worry about in-person victimization may face fewer of these anxious feelings. In contrast, although depressive symptoms may first appear in connection with peer victimization experiences, they may persist despite reductions in victimization due to a lasting impact on teens' biobehavioral functioning in terms of both long-term deficits in reward processing (e.g. Rappaport et al., 2019) and behavioral dysregulation during times of stress (Adrian et al., 2019).

Moreover, pre-pandemic literature links aggression toward peers to depressive symptoms both concurrently and longitudinally and further suggests that directionality favors earlier aggression leading to later depressive symptoms (e.g., Blain-Arcaro \& Vaillancourt, 2017; Dutton \& Karakanta, 2013; Gresham et al., 2016). However, aggression toward peers is seldom directly linked to anxious symptoms in preCOVID literature (e.g., Gresham et al., 2016; Swearer et al., 2001), a pattern of findings that is consistent with the current study. The specificity of the link between peer aggression and depressive symptoms may stem in part from aggressive adolescents' greater tendency to engage in backward-looking rumination about the negative consequences of aggressive actions (such as peer rejection or similar interpersonal failures; Blain-Arcaro \& Vaillancourt, 2017) than in forwardlooking rumination about future aggression.

Other investigators have identified a number of risk factors for adolescents' elevated internalizing response to the pandemic, including COVID-19 exposure (Qi et al., 2020), being left alone during the workday (Chen et al., 2020), female gender (e.g., Chen et al., 2020; Ellis et al., 2020; Zhou et al., 2020), and low socioeconomic status (Magklara et al., 2020; Patrick et al., 2020; Rosen et al., 2020). Our study suggests that loss of positive face-to-face peer relations during the pandemic should be considered an additional risk factor for COVID-19 distress. Furthermore, these findings imply that adolescents who experienced particularly negative peer interactions prior to the pandemic may be at increased risk for rising internalizing symptoms as the social restrictions of COVID-19 are eventually lifted and teens return to in-person interaction. Thus, although the return to "normal life" will likely be welcomed by most teens, we should be vigilant in looking for signs of increasing distress among a subset of adolescents as they return to school, sports, and other social activities.

Beyond the lack of assessment of online and midpandemic peer interactions described above, this study was marked by four additional limitations, each of which may inform directions for future studies. First, our sample was small, community-based, and low-risk, with adolescents reporting fairly low levels of pre-pandemic distress, and thus, our findings may not generalize to more clinical 
samples. Future researchers may want to investigate COVID19 response, and the role of peer relations in that response, in a larger and higher-risk sample experiencing internalizing symptoms at clinically significant levels. Moreover, future pandemic-era longitudinal studies should strive to include greater compensation for participation at later time points to reduce attrition. Second, all measures were collected via self report, an approach which may have inflated relations between constructs. Future researchers should strive to include multiple methodologies in COVID-19 investigations. Third, we do not have data on the degree of isolation participants experienced, the specific physical distancing measures adolescents were required to take, or their compliance with these measures. Other pandemic researchers should strive to collect data on these constructs to provide a more thorough picture of participants' COVID-19 experience. Finally, our mid-pandemic time point was still relatively early in the COVID crisis, and it is not possible to know if our findings would be maintained later in the pandemic.

In conclusion, the current study suggests that adolescents who experienced more difficult interactions with peers prior to the pandemic displayed less change in internalizing symptoms from pre- to mid-pandemic. Rather than simply informing our understanding of adolescents' internalizing response to COVID-19, these findings may increase our insight into the emotional functioning of adolescents who struggle with negative peer relations in non-pandemic times.

Acknowledgements We thank the adolescents and families who participated in this research, as well as the research assistants who helped to collect these data.

\section{Compliance with Ethical Standards}

Conflict of Interest The authors declare that they have no conflict of interest.

\section{References}

Adkins, D. E., Wang, V., \& Elder, G. H. (2009). Structure and stress: Trajectories of depressive symptoms across adolescence and young adulthood. Social Forces, 88, 31-60. https://doi.org/10. 1353/sof.0.0238

Adrian, M., Jenness, J. L., Kuehn, K. S., Smith, M. R., \& McLaughlin, K. A. (2019). Emotion regulation processes linking peer victimization to anxiety and depression symptoms in adolescence. Development and Psychopathology, 31, 999-1009. https://doi.org/10. 1017/s0954579419000543

Bagwell, C. L., \& Bukowski, W. M. (2018). Friendship in childhood and adolescence: Features, effects, and processes. In W. M. Bukowski, B. Laursen, \& K. H. Rubin (Eds.), Handbook of Peer Interactions, Relationships, and Groups (pp. 371-390). Guilford.

Beam, C. R., \& Kim, A. J. (2020). Psychological sequelae of social isolation and loneliness might be a larger problem in young adults than older adults. Psychological Trauma: Theory,
Research, Practice, and Policy, 12(S1), S58. https://doi.org/10. 1037/tra0000774

Blain-Arcaro, C., \& Vaillancourt, T. (2017). Longitudinal associations between depression and aggression in children and adolescents. Journal of Abnormal Child Psychology, 45, 959-970. https://doi. org/10.1007/s10802-016-0204-2

Boulton, M. J., Trueman, M., \& Murray, L. (2008). Associations between peer victimization, fear of future victimization and disrupted concentration on class work among junior school pupils. British Journal of Educational Psychology, 78, 473-489. https:// doi.org/10.1348/000709908X320471

Chen, F., Zheng, D., Liu, J., Gong, Y., Guan, Z., \& Lou, D. (2020). Depression and anxiety among adolescents during COVID-19: A cross-sectional study. Brain, Behavior, and Immunity, 88, 36-38. https://doi.org/10.1016/j.bbi.2020.05.061

Dutton, D. G., \& Karakanta, C. (2013). Depression as a risk marker for aggression: A critical review. Aggression and Violent Behavior, 18, 310-319. https://doi.org/10.1016/j.avb.2012.12.002

Ellis, W. E., Dumas, T. M., \& Forbes, L. M. (2020). Physically isolated but socially connected: Psychological adjustment and stress among adolescents during the initial COVID-19 crisis. Canadian Journal of Behavioural Science, 52, 177-187. https://doi.org/10. 1037/cbs0000215

Forbes, M. K., Fitzpatrick, S., Magson, N. R., \& Rapee, R. M. (2019). Depression, anxiety, and peer victimization: Bidirectional relationships and associated outcomes transitioning from childhood to adolescence. Journal of Youth and Adolescence, 48, 692-702. https://doi.org/10.1007/s10964-018-0922-6

Gadassi Polack, R., Sened, H., Aube, S., Zhang, A., Joormann, J., \& Kober, H. (2021, May 28). Connections during crisis: Adolescents' social dynamics and mental health during COVID-19. PsyArXiv Preprints. https://doi.org/10.1037/dev0001211

Gini, G., Card, N. A., \& Pozzoli, T. (2018). A meta-analysis of the differential relations of traditional and cyber-victimization with internalizing problems. Aggressive Behavior, 44, 185-198. https:// doi.org/10.1002/ab.21742

Gresham, D., Melvin, G. A., \& Gullone, E. (2016). The role of anger in the relationship between internalizing symptoms and aggression in adolescents. Journal of Child and Family Studies, 25, 2674 2682. https://doi.org/10.1007/s10826-016-0435-4

Hall-Lande, J. A., Eisenberg, M. E., Christenson, S. L., \& NeumarkSztainer, D. (2007). Social isolation, psychological health, and protective factors in adolescence. Adolescence, 42, 265286. https://pubmed.ncbi.nlm.nih.gov/17849936/

Hawker, D. S. J., \& Boulton, M. J. (2000). Twenty years' research on peer victimization and psychosocial adjustment: A meta-analytic review of cross-sectional studies. Journal of Child Psychology and Psychiatry, 41, 441-455. https://pubmed.ncbi.nlm.nih.gov/10836674/

Hossain, M. M., Sultana, A., \& Purohit, N. (2020). Mental health outcomes of quarantine and isolation for infection prevention: A systematic umbrella review of the global evidence. Epidemiology and Health, e2020038. https://doi.org/10.4178/epih.e2020038

Kievit, R. A., Brandmaier, A. M., Ziegler, G., van Harmelen, A. L., de Mooij, S. M. M., Moutoussis, M., Goodyer, I. M., \& Dolan, R. J. (2018). Developmental cognitive neuroscience using latent change score models: A tutorial and applications. Developmental Cognitive Neuroscience, 33, 99-117. https://doi.org/10.1016/j.den.2017.11.007

Kovacs, M. (2011). Children's Depression Inventory 2. North Tonawanda, NY: Multi-Health Systems.

Little, T. D., Jones, S. M., Henrich, C. C., \& Hawley, P. H. (2003). Detangling the "whys" from the "whats" of aggressive behavior. International Journal of Behavioral Development, 27, 122-133. https://doi.org/10.1080/01650250244000128

Liu, C. H., Zhang, E., Wong, G. T. F., Hyun, S., \& Hahm, H. C. (2020). Factors associated with depression, anxiety, and PTSD 
symptomatology during the COVID-19 pandemic: Clinical implications for US young adult mental health. Psychiatry Research, 290, 113172. https://doi.org/10.1016/j.psychres.2020.113172

Loades, M. E., Chatburn, E., Higson-Sweeney, N., Reynolds, S., Shafran, R., Brigden, A., Linney, C., \& Crawley, E. (2020). Rapid systematic review: The impact of social isolation and loneliness on the mental health of children and adolescents in the context of Covid-19. Journal of the American Academy of Child and Adolescent Psychiatry, 59, 1218-1239. https://doi.org/10.1016/j.jaac.2020.05.009

Magklara, K., Lazaratou, H., Barbouni, A., Poulas, K., Farsalinos, K., \& Coronavirus Greece Research Group. (2020). Impact of COVID-19 pandemic and lockdown measures on mental health of children and adolescents in Greece. medRxiv. https://doi.org/ $10.1101 / 2020.10 .18 .20214643$

Malkus, N., \& Christensen, C. (2020). School District Responses to the COVID-19 Pandemic: Round 3, Plans for a Remote Finish. American Enterprise Institute.

March, J. S., Parker, J. D. A., Sullivan, K., Stallings, P., \& Conners, C. K. (1997). The Multidimensional Anxiety Scale for Children (MASC): Factor structure, reliability, and validity. Journal of the American Academy of Child and Adolescent Psychiatry, 36, 554-565. https://doi.org/10.1097/00004583-199704000-00019

McArdle, J. J. (2009). Latent variable modeling of differences and changes with longitudinal data. Annual Review of Psychology, 60, 577-605. https://doi.org/10.1146/annurev.psych.60.110707.163612

McDougall, P., \& Vaillancourt, T. (2015). Long-term adult outcomes of peer victimization in childhood and adolescence. American Psychologist, 70, 300-310. https://doi.org/10.1037/a0039174

Morrow, M. T., Hubbard, J. A., Bookhout, M. K., Grassetti, S. N., Docimo, M. A., \& Swift, L. E. (2021). Development and validation of the Forms of Peer Victimization Scale. Merrill-Palmer Quarterly, 67, 23-55. https://doi.org/10.13110/merrpalmquar1982. 67.1.0023

Muthén, L. K., \& Muthén, B. O. (1998-2017). Mplus User's Guide. Eighth Edition. Los Angeles, CA: Muthén \& Muthén.

Nelemans, S. A., Hale, W. W., Branje, S. J., Raajmakers, Q. A., Frijns, T., van Lier, P. A., \& Meeus, W. H. (2014). Heterogeneity in development of adolescent anxiety disorder symptoms in an 8-year longitudinal community study. Development and Psychopathology, 26, 181-202. https://doi.org/10.1017/S0954579413000503

Ohannessian, C. M., Milan, S., \& Vannucci, A. (2017). Gender differences in anxiety trajectories from middle to late adolescence. Journal of Youth and Adolescence, 46, 826-839. https://doi.org/ 10.1007/s10964-016-0619-7

Oosterhoff, B., Palmer, C. A., Wilson, J., \& Shook, N. (2020). Adolescents' motivations to engage in social distancing during the COVID-19 pandemic: Associations with mental and social health. Journal of Adolescent Health., 67, 179-185. https://doi.org/10. 1016/j.jadohealth.2020.05.004

Patrick, S. W., Henkhaus, L. E., Zickafoose, J. S., Lovell, K., Halvorson, A., Loch, S., \& Davis, M. M. (2020). Well-being of parents and children during the COVID-19 pandemic: A national survey. Pediatrics, 146, e2020016824. https://doi.org/10.1542/peds.2020-016824

Qi, M., Zhou, S. J., Guo, Z. C., Zhang, L. G., Min, H. J., Li, X. M., \& Chen, J. X. (2020). The effect of social support on mental health in Chinese adolescents during the outbreak of COVID-19. Journal of Adolescent Health, 67, 514-518. https://doi.org/10.1016/j. jadohealth.2020.07.001

Qualter, P., \& Munn, P. (2002). The separateness of social and emotional loneliness in childhood. Journal of Child Psychology and Psychiatry, 43, 233-244. https://doi.org/10.1111/1469-7610.00016

Rappaport, B. I., Hennefield, L., Kujawa, A., Arfer, K. B., Kelly, D., Kappenman, E. S., \& Barch, D. M. (2019). Peer victimization and dysfunctional reward processing: ERP and behavioral responses to social and monetary rewards. Frontiers in Behavioral Neuroscience, 13, 120. https://doi.org/10.3389/fnbeh.2019.00120

Reijntjes, A., Kamphuis, J. H., Prinzie, P., \& Telch, Ml. J. (2010). Peer victimization and internalizing problems in children: A meta-analysis of longitudinal studies. Child Abuse and Neglect, 34, 244-252. https://doi.org/10.1016/j.chiabu.2009.07.009

Rodman, A. M., Rosen, M. L., Kasparek, S. W., Mayes, M., Lengua, L., McLaughlin, K. A., \& Meltzoff, A. N. (2021, March 4). Social behavior and youth psychopathology during the COVID-19 pandemic: A longitudinal study. PsyArXiv Preprints. https://doi.org/ 10.31234/osf.io/68zvg

Rosen, Z., Weinberger-Litman, S. L., Rosenzweig, C., Rosmarin, D. H., Muennig, P., Carmody, E. R., Rao, S. T., \& Litman, L. (2020, April 14). Anxiety and distress among the first community quarantined in the US due to COVID-19: Psychological implications for the unfolding crisis. PsyArXiv Preprints. https://doi.org/10.31234/osf.io/7eq8c

Rothenberg, W. A., Di Giunta, L., Lansford, J. E., Lunetti, C., Fiasconaro, I., Basili, E., \& Cirimele, F. (2019). Daily associations between emotions and aggressive and depressive symptoms in adolescence: The mediating and moderating role of emotion dysregulation. Journal of Youth and Adolescence, 48, 2207-2221. https://doi.org/10.1007/ s10964-019-01071-6

Rubin, K. H., Bowker, J. C., Barstead, M. G., \& Coplan, R. J. (2018). Avoiding and withdrawing from the peer group. In W. M. Bukowski, B. Laursen, \& K. H. Rubin (Eds.), Handbook of Peer Interactions, Relationships, and Groups (pp. 322-348). Guilford.

Salmivalli, C., \& Peets, K. (2018). Bullying and victimization. In W. M. Bukowski, B. Laursen, \& K. H. Rubin (Eds.), Handbook of Peer Interactions, Relationships, and Groups (pp. 302-321). Guilford.

Schubert, K. O., Clark, S. R., Van, L. K., Collinson, J. L., \& Baune, B. T. (2017). Depressive symptom trajectories in late adolescence and early adulthood: A systematic review. Australian and New Zealand Journal of Psychiatry, 51, 477-499. https://doi.org/10. 1177/0004867417700274

Swearer, S. M., Song, S. Y., Cary, P. T., Eagle, J. W., \& Mickelson, W. T. (2001). Psychosocial correlates of bullying and victimization: The relationship between depression, anxiety, and bully/victim status. Journal of Emotional Abuse, 2, 95-121. https://doi.org/ 10.1300/J135v02n02_07

Vitaro, F., Boivin, M., \& Poulin, F. (2018). The interface of aggression and peer relations in childhood and adolescence. In W. M. Bukowski, B. Laursen, \& K. H. Rubin (Eds.), Handbook of Peer Interactions, Relationships, and Groups (pp. 284-301). Guilford.

Vuijk, P., van Lier, P. A., Crijnen, A. A., \& Huizink, A. C. (2007). Testing sex-specific pathways from peer victimization to anxiety and depression in early adolescents through a randomized intervention trial. Journal of Affective Disorders, 100, 221-226. https://doi.org/ 10.1016/j.jad.2006.11.003

Xie, X., Xue, Q., Zhou, Y., Zhu, K., Liu, Q., Zhang, J., \& Song, R. (2020). Mental health status among children in home confinement during the coronavirus disease 2019 outbreak in Hubei Province, China. JAMA Pediatrics, 171, 898-900. https://doi.org/10.1001/ jamapediatrics.2020.1619

Zhou, S. J., Zhang, L. G., Wang, L. L., Guo, Z. C., Wang, J. Q., Chen, J. C., \& Chen, J. X. (2020). Prevalence and socio-demographic correlates of psychological health problems in Chinese adolescents during the outbreak of COVID-19. European Child \& Adolescent Psychiatry, 29, 749-758. https://doi.org/10.1007/s00787-020-01541-4

Publisher's Note Springer Nature remains neutral with regard to jurisdictional claims in published maps and institutional affiliations. 